\title{
Avaliação de atividade de ensino, pesquisa e extensão em vigilância do desenvolvimento infantil: a perspectiva de graduandos em terapia ocupacional*
}

\section{Teaching activity, research and extension evaluation in surveillance of child development: the perspective of occupational therapy undergraduate students}

\author{
Patrícia Carla de Souza Della Barba ${ }^{3,4}$, Aline Gonçalves', Bárbara Aniceto ${ }^{1}$,

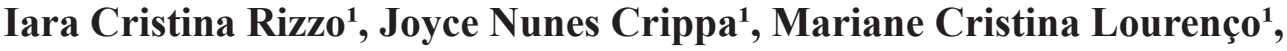 \\ Nathalia Abreu dos Santos', Thais Tiemi Nishiama ${ }^{1}$, Andressa Fernanda Jóia ${ }^{2}$, \\ Raquel Cristina Pinheiro², Bruna Pereira Ricci Marini², Claudia Maria Simões \\ Martinez $^{3}$, Regina Helena Vitale Torkomian Joaquim ${ }^{3}$
}

http://dx.doi.org/10.11606/issn.2238-6149.v26i2p274-280

\begin{abstract}
Della Barba PCS, Gonçalves A, Aniceto B, Rizzo IC, Crippa JN, Lourenço MC, Santos NA, Nishiama TT, Jóia AF, Pinheiro RC, Marini BPR, Martinez CSM, Joaquim RHVT. Avaliação de atividade de ensino, pesquisa e extensão em vigilância do desenvolvimento infantil: a perspectiva de graduandos em terapia ocupacional. Rev Ter Ocup Univ São Paulo. 2015 maio-ago.;26(2):274-80.
\end{abstract}

RESUMO: A Atividade Curricular de Integração Ensino, Pesquisa e Extensão (ACIEPE) "Desenvolvimento infantil de zero a seis anos e a atuação da Terapia Ocupacional", oferecida por docentes do curso de Terapia Ocupacional da Universidade Federal de São Carlos, de março a setembro de 2013, teve por objetivo criar um diálogo entre educadores da rede municipal de educação infantil e graduandos sobre temas que envolvem a promoção do desenvolvimento infantil no cotidiano familiar e escolar. Trata-se de um relato de experiência que descreve a avaliação dos estudantes acerca dos conteúdos abordados nesta ACIEPE discutindo as implicações desta atividade na formação.
Estudo investigativo descritivo, de abordagem qualitativa, com aplicação de questionário para dezenove alunos que participaram da ACIEPE. Destacam-se ganhos: na vivência interdisciplinar; no contato com os educadores; na produção de intervenções e no aprofundamento sobre as práticas de Terapia Ocupacional. Estudantes também identificam a experiência como desafiadora e afirmam ter obtido maior aprofundamento sobre as possibilidades da prática de Terapia Ocupacional no contexto da educação infantil, aprimorando seus conhecimentos acerca do processo de vinculação tanto com as crianças quanto com os educadores.

DESCRITORES: Vigilância; Desenvolvimento infantil; Terapia Ocupacional/educação; Educação infantil; Educação superior; Ocupações em saúde/educação; Estudantes.

Della Barba PCS, Gonçalves A, Aniceto B, Rizzo IC, Crippa JN, Lourenço MC, Santos NA, Nishiama TT, Jóia AF, Pinheiro RC, Marini BPR, Martinez CSM, Joaquim RHVT. Teaching activity, research and extension evaluation in surveillance of

\footnotetext{
* O trabalho é um dos produtos da Atividade Integrada Curricular de Ensino Pesquisa e Extensão intitulada "Desenvolvimento infantil de zero a seis anos e a atuação da Terapia Ocupacional", desenvolvida pelo departamento de Terapia Ocupacional da UFSCar.

1. Estudantes de graduação do curso de Terapia Ocupacional da Universidade Federal de São Carlos, participantes da equipe da ACIEPE. E-mails: alinegoncalves603@gmail.com, ba_aniceto@live.com, iara_rizzo@hotmail.com, joycecrippa@hotmail.com, marianec. lourenco@gmail.com, nathalia.yoona@gmail.com, thatanishiama@gmail.com.

2. Terapeutas ocupacionais graduadas pela Universidade Federal de São Carlos, colaboradoras da ACIEPE. E-mails: dessa_joia@hotmail. com, raquelpinheiro.to@gmail.com, brunamarini_to@yahoo.com.br.

3. Professores doutores, docentes adjuntos do curso de Terapia Ocupacional da Universidade Federal de São Carlos. E-mails: claudia@ufscar.br, regin@ufscar.br.

4. Coordenadora da ACIEPE (Atividade Integrada Curricular de Ensino Pesquisa e Extensão). E-mail: patriciadellabarba@yahoo.com.br. Endereço para correspondência: Prof ${ }^{a}$. Dra. Patrícia CS Della Barba. Departamento de Terapia Ocupacional da UFSCar. Rodovia Washington Luís, km 235 - SP-310. São Carlos, São Paulo, Brasil. CEP: 13565-905. E-mail: patriciadellabarba@yahoo.com.br.
} 
child development: the perspective of occupational therapy undergraduate students. Rev Ter Ocup Univ São Paulo. 2015 May-Aug.;26(2):275-80.

ABSTRACT: The Curricular Activity of Integration between Education, Research and Extension (ACIEPE) "Child development from birth to six years and the work of Occupational Therapy", offered by the faculty of Occupational Therapy of Federal University of São Carlos between March and September 2013, aimed to create a dialogue between educators of the municipal network of early childhood education and undergraduate students on issues involving the promotion of child development in family and school routines. This is an experience report describing the students' evaluation of the content covered in this ACIEPE,

\section{INTRODUÇÃO}

$\mathrm{A}$ Atividade Curricular de Integração Ensino, Pesquisa e Extensão (ACIEPE) é uma experiência educativa, cultural e científica que envolve professores, técnicos e alunos, com o intuito de estimular o relacionamento de diferentes segmentos da sociedade por meio da articulação do ensino, pesquisa e extensão ${ }^{1}$.

A ACIEPE "Desenvolvimento infantil de zero a seis anos e a atuação da Terapia Ocupacional", oferecida por docentes do curso de Terapia Ocupacional da UFSCar, teve como proposta criar o diálogo entre educadores de creches, pertencentes à rede municipal de educação infantil, e graduandos do curso de Terapia Ocupacional da UFSCar sobre temas que levam à promoção do desenvolvimento infantil no cotidiano da família e da escola.

A ACIEPE, depois de aprovada pela pró-reitoria de extensão, foi divulgada à comunidade tanto interna quanto externa da universidade. Após a divulgação, foram realizadas as inscrições dos educadores por demanda espontânea. Os estudantes o fizeram por meio do sistema de matrículas em disciplinas da universidade. Participaram da atividade 49 educadores de creches públicas da cidade de São Carlos e região e vinte estudantes dos períodos iniciais de graduação em Terapia Ocupacional. Foram ministradas sete aulas teórico-práticas que abordaram os temas: vigilância do desenvolvimento; desenvolvimento emocional e apego; desenvolvimento psicomotor, visual e cognitivo; a importância do brincar para o desenvolvimento infantil e os fatores de risco e proteção para a saúde mental da criança.

O cronograma das aulas foi divulgado aos participantes, que deveriam enviar materiais (fotos, vídeos e depoimentos) referentes ao cotidiano dos Centros Municipais de Educação Infantil (CEMEIS). discussing the implications of this activity in education. It is a descriptive investigative study of qualitative approach with the application of a questionnaire to nineteen students who participated in the ACIEPE. We highlight gains in the interdisciplinary experience, in communication with teachers, in the production of interventions and in increasing knowledge about Occupational Therapy practices. Students also identify the experience as challenging and claim to have obtained greater deepening of the possibilities of Occupational Therapy practice in the context of early childhood education, improving their knowledge about the binding process with both children and teachers.

KEYWORDS: Surveillance; Child development; Occupational therapy; Childhood education; Education, higher; Health occupations/education; Students.

Foram realizados encontros semanais, no período noturno, com duração de três horas, divididos em duas etapas. Na primeira, foram ministradas aulas dialogadas, com conteúdo preparado previamente pelos docentes coordenadores da ACIEPE, bem como por convidados considerados especialistas nos temas abordados. O conteúdo das aulas contemplou o material enviado pelos participantes, que foram incorporados e mesclados ao conteúdo teórico de forma dinâmica. Na segunda etapa, os participantes planejaram e desenvolveram um projeto de intervenção nas CEMEIs nos quais estavam inseridos, buscando responder às demandas por eles identificadas nas CEMEIs. Houve a divisão dos educadores e alunos participantes em subgrupos e, em momentos extraclasse, esses se encontraram nas próprias CEMEIs para efetivarem juntos as intervenções propostas.

Os projetos foram desenvolvidos por meio de uma a três sessões de intervenção, no período diurno, acompanhando o horário de trabalho dos educadores, e variaram entre intervenções direcionadas à criança, à capacitação de educadores e pais e intervenções ambientais.

A atividade propiciou aos participantes a oportunidade de trocar conhecimentos, objetivando a descoberta e experimentação de alternativas de solução e encaminhamento de problemas, na perspectiva da produção de conhecimentos na área. Como ensino, a atividade visou à oportunidade dos estudantes vivenciarem o conhecimento sobre o desenvolvimento infantil, no próprio contexto da educação e por meio da troca de experiências com os educadores, fora do ambiente da Universidade. Neste sentido, a atividade foi ao encontro do que é preconizado no curso de graduação em Terapia Ocupacional da UFSCar, no qual há incentivo ao aluno, desde o primeiro ano, a participar ativamente de seu 
processo de aprendizagem. O currículo é baseado nas metodologias ativas de aprendizagem em que o contato com a clientela e com as situações reais ou simuladas da prática profissional é fundamental para formar profissionais capazes de planejar e gerir serviços e construir novos modelos de cuidado ${ }^{2}$.

O principal tema abordado no projeto foi a promoção do desenvolvimento infantil na faixa etária de 0 a 6 anos. Considerando que o processo de desenvolvimento da criança está associado à carga genética, às condições de saúde e às influências familiares, ambientais, culturais e sociais ${ }^{3}$, as seguintes premissas nortearam a definição dos subtemas propostos e as estratégias metodológicas empregadas:

- A escola tem potencial para influir no desenvolvimento das crianças, mas é necessário investir em sua qualidade. É preciso que na pré-escola sejam desenvolvidas atividades de estímulo às crianças e que permitam promoção do desenvolvimento saudável e compatível com o que se espera em cada faixa etária ${ }^{4,5}$;

- O monitoramento do desenvolvimento da criança deve ser realizado em seu contexto natural e inclui atividades e oportunidades que visam incentivar a aprendizagem e o desenvolvimento ${ }^{6}$.

- A Terapia Ocupacional pode contribuir na formação de educadores infantis na perspectiva de favorecer o desenvolvimento das crianças nos contextos de vida diária, particularmente o da creche ${ }^{7-10}$;

- A visão integral e compartilhada da criança por profissionais da educação e da saúde pode contribuir para a detecção precoce de fatores de risco e para a promoção da qualidade das interações e do ambiente em que estão inseridas. Essa detecção se constitui numa ação primordial no contexto da vigilância do desenvolvimento pelas possibilidades de minimizar efeitos do risco à criança em momento oportuno e impedir que possíveis déficits se instalem ${ }^{8}$;

- As atividades de vida diária e as brincadeiras constituem-se em oportunidades de desenvolvimento infantil. A ocupação é o foco da prática do terapeuta ocupacional, e este deve analisar as atividades cotidianas em que o indivíduo espera participar; desta forma, incentivar a participação nas atividades típicas da infância deve ser o maior foco dos terapeutas ocupacionais que trabalham com essa população ${ }^{11}$.

Espera-se que o aluno de graduação em Terapia Ocupacional: torne-se competente para analisar e acompanhar o desenvolvimento infantil e as atividades típicas da infância; desenvolva-se como profissional capaz de ativar mecanismos protetivos; atue na vigilância do desenvolvimento, sob a perspectiva de minimizar possíveis riscos; promova intervenções no ambiente escolar e orientações a educadores e familiares ${ }^{12,13}$.

A partir do diálogo estabelecido entre educadores da rede municipal de educação infantil e graduandos do curso de Terapia Ocupacional da UFSCar, o objetivo deste artigo é avaliar a atividade de extensão ACIEPE, sob a perspectiva dos estudantes, bem como apontar sua contribuição para a formação em Terapia Ocupacional.

\section{METODOLOGIA}

O estudo descritivo de abordagem qualitativa desenvolveu os seguintes procedimentos: aplicação de questionário para alunos que participaram da ACIEPE, contendo oito perguntas abertas e duas perguntas fechadas, que abordaram a opinião dos participantes quanto ao conteúdo e importância da temática ministrada, percepções com relação ao trabalho junto aos educadores, sugestões e dificuldades encontradas durante as aulas.

Os questionários foram enviados aos participantes. A análise dos dados ocorreu por meio da análise de conteúdo, reunindo-se as temáticas das respostas dos participantes $^{14}$.

\section{RESULTADOS}

Dos vinte questionários enviados, dezenove foram respondidos e analisados, o que resultou em quatro categorias de análise: estratégia da ACIEPE; contato do estudante com o educador; relevância do conhecimento e; conhecimento e atuação da Terapia Ocupacional na creche, que serão apresentadas a seguir.

\section{Estratégia da ACIEPE}

Os estudantes analisaram aspectos referentes a: estruturação das aulas, temáticas e participação dos palestrantes e a importância da ACIEPE no processo de formação profissional. Os resultados apontam predomínio de avaliações positivas, sendo que os alunos ressaltaram: o bom planejamento; a importância das temáticas abordadas 
para a compreensão do processo de desenvolvimento infantil; a propriedade dos palestrantes em relação às temáticas e a relevância da atividade na formação profissional:

Avalio positivamente, pois pude refletir muito acerca de um tema que me interessa, além de absorver e aprender conteúdos muito relevantes, com todos os temas. A estrutura das aulas, que permitia conversas, reflexões e dúvidas, puderam contribuir com meu conhecimento, além de oferecer muitos aprendizados. Os palestrantes se mostraram disponiveis e expuseram os temas de forma muito compreensiva. (S9)

\section{Contato do estudante com o educador}

Os estudantes também avaliaram seu contato com o educador no processo de aulas, grupos e intervenções nas CEMEIs. Foram observados resultados referentes à importância do trabalho interdisciplinar e sua relevância para a troca de conhecimentos e vivências.

No questionário respondido por eles constava a pergunta aberta sobre como qualificavam a relação estabelecida durante a atividade. As respostas dos estudantes foram analisadas quanto ao seu conteúdo, e estabeleceu-se categorias, de acordo com Bardin ${ }^{14}$. A classificação dessa interação é apresentada no Gráfico 1.

A vivência nesta atividade foi classificada pela maioria dos estudantes ( $\mathrm{N}=15$ ou $79 \%$ ) como "enriquecedora" no que diz respeito ao contato com o educador, pois acreditam que os educadores contribuíram com suas experiências de trabalho na CEMEI durante as atividades desenvolvidas:

O trabalho conjunto com as educadoras foi muito produtivo, pois elas relataram várias experiências e dificuldades que vivem na CEMEI, o que foi muito rico para a nossa formação. (S7)

[...] foi bem enriquecedor porque eu consegui aprender muita coisa e ficar mais consciente a partir de situações reais contadas pelas educadoras e até vivenciadas nas visitas. (S15)

Nove alunos (47\%) consideraram a experiência "desafiadora", pois, devido ao curso de graduação ser em período integral, tinham tempo limitado para se dedicar às tarefas da ACIEPE. O horário das aulas (noturno) e o planejamento e execução dos projetos de intervenção eram realizados em período extraclasse:

É desafiador e exaustivo devido às horas de pesquisa e o horário das aulas. (S2)

Achei que foi bem desafiador porque na divisão feita, o nosso grupo ficou com educadoras que eram de São Carlos e também de creches diferentes, então isso demandou um trabalho maior [...] (S15)

Gráfico 1 - Classificação do trabalho em conjunto com os educadores

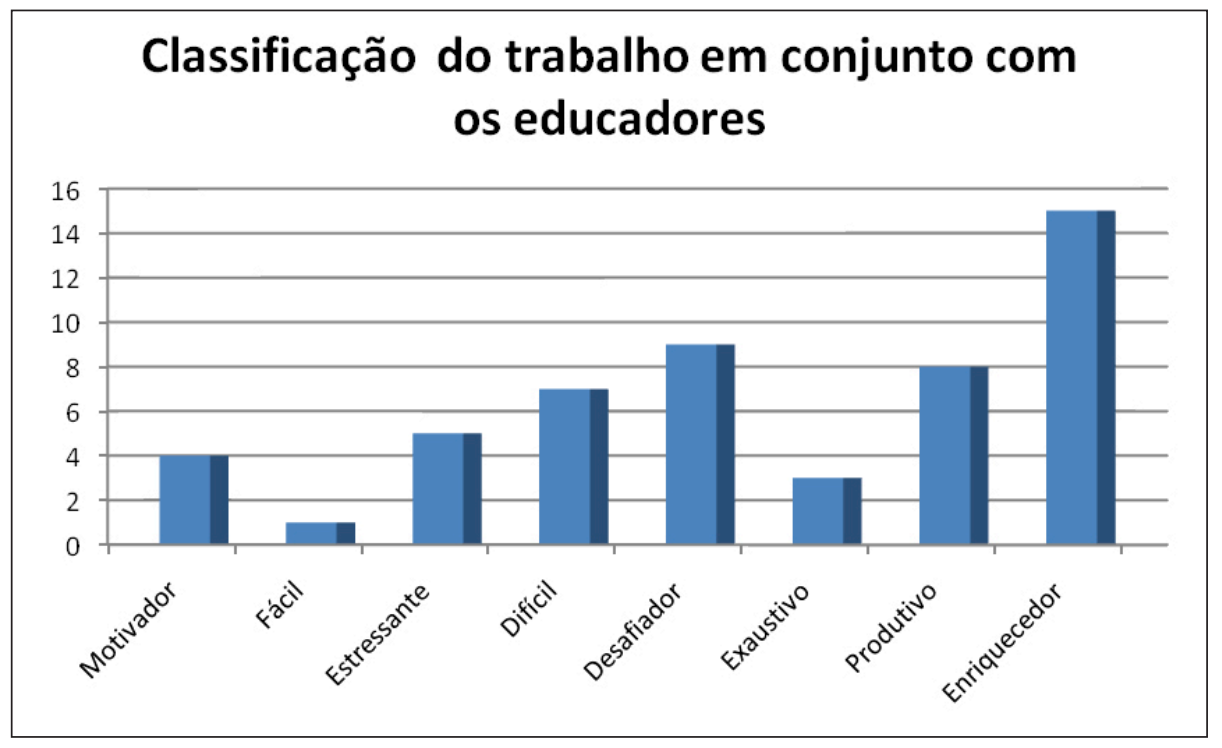


Della Barba PCS, et al. Avaliação de atividade de ensino, pesquisa e extensão. Rev Ter Ocup Univ São Paulo. 2015 maio/ago.;26(2):275-80.

Oito estudantes (42\%) avaliaram a atividade como "produtiva", relatando que, mesmo tendo se reunido poucas vezes com os educadores, conseguiram elaborar projetos de intervenção interessantes nas CEMEIs.

Quatro alunos (21\%) consideraram a experiência "motivadora" pela ação conjunta com o educador, pelo conhecimento do mesmo e sua dedicação em aprimorar-se:

Durante muitos encontros o trabalho em conjunto foi motivador e enriquecedor, pois são pessoas que já vivenciam e trabalham com crianças e na prática veem o que foi trabalhado em sala. (S10)

Cinco estudantes $(26 \%)$ classificaram a atividade como "estressante", explicando a divisão das tarefas do grupo:

[...] elaborar o trabalho foi muito estressante, pois as alunas ficaram com a maioria de coisas para fazer, refazendo várias vezes para se adequar às normas e às referências. (S9)

Sete estudantes $(37 \%)$ classificaram a atividade como "difícil", justificando que, apesar da aprendizagem proporcionada pela interação aluno-educador, os momentos em grupo poderiam ser mais bem explorados.

\section{Relevância do conhecimento}

Os alunos apontaram a importância da ACIEPE para desenvolver conhecimento a respeito da vigilância do desenvolvimento e a educação infantil. Enfatizaram o aprendizado de novos conceitos, como a atuação do terapeuta ocupacional nessa área:

A ACIEPE auxiliou muito no conhecimento sobre a Vigilância do desenvolvimento e Desenvolvimento Infantil, principalmente por eu estar no primeiro ano. Me deu muita base para aprofundar meus estudos sobre esse tema. (S2)

Foi proposto no questionário que os estudantes avaliassem os conteúdos apresentados nas aulas de acordo com sua relevância: Muito grande, Grande, Razoável e/ ou Pouco.

Como demonstrado na Tabela 1 , os temas Vigilância do desenvolvimento, Desenvolvimento cognitivo, Desenvolvimento visual, Desenvolvimento psicossocial/Teoria do apego e a Importância do brincar foram avaliados como de grande relevância pelos estudantes.

Tabela 1 - Classificação da relevância das temáticas apresentadas durante os encontros

\begin{tabular}{|c|c|c|c|c|c|}
\hline \multicolumn{6}{|c|}{ Classificação da relevância das temáticas apresentadas durante os encontros } \\
\hline & Muito grande & Grande & Razoável & Pouco & Não respondeu \\
\hline Vigilância do desenvolvimento & $57,89 \%$ & $36,84 \%$ & $5,26 \%$ & $0 \%$ & $0 \%$ \\
\hline Desenvolvimento cognitivo & $52,63 \%$ & $47,37 \%$ & $0 \%$ & $0 \%$ & $0 \%$ \\
\hline Desenvolvimento visual & $52,63 \%$ & $36,84 \%$ & $5,26 \%$ & $0 \%$ & $5,26 \%$ \\
\hline Desenvolvimento psicossocial/ Teoria do apego & $36,84 \%$ & $63,16 \%$ & $0 \%$ & $0 \%$ & $0 \%$ \\
\hline Saúde mental & $42,1 \%$ & $36,84 \%$ & $21,05 \%$ & $0 \%$ & $0 \%$ \\
\hline Importância do brincar & $63,16 \%$ & $31,58 \%$ & $0 \%$ & $0 \%$ & $5,26 \%$ \\
\hline
\end{tabular}

\section{Conhecimento e atuação da Terapia Ocupacional na creche}

Notou-se que a ACIEPE colaborou de forma significativa para o conhecimento sobre a atuação da Terapia Ocupacional no campo do desenvolvimento infantil, proporcionando aos alunos envolvimento com o tema e estimulando-os a prosseguir com os estudos nessa área:

Proporciona aos alunos o conhecimento de um campo muito importante de atuação da Terapia Ocupacional. (S14).
Não tinha muito contato com o desenvolvimento infantil e me senti estimulada a continuar estudando sobre. (S8).

Ao serem questionados a respeito da atuação da terapia ocupacional em contexto escolar, pode-se observar que a maioria dos alunos tinha conhecimento dessa possibilidade, exceto um estudante que relata conhecer muito pouco e outro que diz não ter tido conhecimento. Dois relataram ter conhecimento somente em casos voltados para necessidades especiais, em instituições como a APAE, por exemplo, ou então em escolas particulares: 
Sabia que poderíamos trabalhar em instituições como a APAE, mas em escolas regulares não tinha conhecimento. (S17)

Dez estudantes relataram que sabiam superficialmente sobre a atuação da Terapia Ocupacional no contexto escolar, tendo tido acesso a informações por meio de reportagens, matérias da faculdade, professores de graduação e matéria na revista CREFITO-SP.

A maioria deles relatou que a ACIEPE contribuiu para um maior aprofundamento sobre as possibilidades de prática da terapia ocupacional nesse contexto:

Eu conhecia, porém de maneira superficial. Por isso a ACIEPE contribuiu para que eu conhecesse um pouco melhor essa possibilidade, que, aliás, gostei muito e achei muito importante e necessária a presença de um terapeuta ocupacional na escola. (S5)

Foi colocada também pelos estudantes a importância de o terapeuta ocupacional estar atento aos aspectos relacionados ao desenvolvimento infantil para poder contribuir tanto junto às famílias como aos educadores.

\section{CONSIDERAÇÕES FINAIS}

A partir do diálogo estabelecido entre educadores da rede municipal de educação infantil e estudantes de graduação do curso de Terapia Ocupacional da UFSCar,

\section{REFERÊNCIAS}

1. Universidade Federal de São Carlos. PROEX Pró-reitoria de Extensão. ACIEPES [citado 18 dez. 2013]. Disponível em: http://www.proex.ufscar.br/site/menu-1/aciepes.

2. Della Barba PCS, Silva RF, Joaquim RHVT, Brito CMD. Formação inovadora em Terapia Ocupacional. Rev Interface - Comum Saúde Educ (Botucatu). 2012;16(42):545-58. DOI: http://dx.doi.org/10.1590/S1414-32832012000300019.

3. Pinto EB. O desenvolvimento do bebê prematuro no primeiro ano de vida. Psicol Reflex Crít. 2009;22(1):76-85. DOI: http://dx.doi.org/10.1590/S0102-79722009000100011.

4. Klein R. A pré-escola no Brasil. Rev Eletrônica Ibero-americana Calidad Eficácia Cambio Educ. 2007;5(2):273-88. Disponível em: http://www.rinace.net/arts/vol5num2e/art19.pdf. constituído na vivência da ACIEPE, este artigo objetivou avaliar uma atividade de extensão na formação do estudante, a partir de sua perspectiva. Constatou-se a importância dessa atividade integrada para a formação do aluno de Terapia Ocupacional em vigilância do desenvolvimento e atuação na educação infantil.

$\mathrm{O}$ relato evidenciou ganhos identificados pelos estudantes especialmente nos aspectos de planejamento da atividade, conteúdos abordados e oportunidade de aquisição de novos conceitos. Além disso, o trabalho interdisciplinar e o conhecimento acerca da realidade da educação infantil foram destacados, confirmando a pertinência da interação de conhecimentos advindos do campo da saúde e educação infantil, podendo trazer ganhos para a detecção precoce de fatores de risco para problemas do desenvolvimento infantil.

O terapeuta ocupacional tem atuado no campo da intervenção precoce, buscando a promoção do desenvolvimento infantil e a prevenção de alterações do desenvolvimento na infância. Dessa maneira, tem condições de estender suas intervenções para o ambiente da educação infantil, analisando os recursos e o contexto para realizar modificações ou adaptações que suscitem soluções práticas para os problemas existentes ${ }^{8,10}$.

Constata-se, com tal atividade, a possibilidade de preparar o futuro profissional para o mercado de trabalho e a aquisição de competências para atuar em equipes diversas e novos territórios para a Terapia Ocupacional, bem como a construção de novos modelos de cuidado.
5. Mastroianni ECQ, Bofi TC, Carvalho AC, Saita LS, Cruz, MLS. Perfil do desenvolvimento motor e cognitivo de crianças com idade entre zero e um ano matriculadas nas creches públicas da rede municipal de educação de Presidente Prudente. Rev Ibero-Americana Est Educ. 2007;2(1):178-88. Disponível em: file://C:/Users/Administrador/Downloads/ perfildodesenvolvimentomotor.pdf.

6. Dunst C, Bruder MB. Valued outcomes of service coordination, earlyinterventionandnaturalenvironments. ExceptionalChildren. 2002;68(3):361-75. Disponível em: http://www.uconnucedd. org/pdfs/projects/rtc/ValuedOutcomesServCoord-2002.pdf.

7. Martinez CMS, Della Barba PCS, Paixão PC, Rodrigues DS. Desenvolvimento de bebês: atividades cotidianas e interação com o educador. 2a ed. São Carlos: EdUFSCar; 2010. 
8. Neófiti CC, Martinez CMS. Provisão de suporte informativo aos educadores de creche: contribuições da Terapia Ocupacional no desenvolvimento de bebês na faixa etária de 0-1 ano. In: XIV Congresso de Iniciação Científica da Universidade Federal de São Carlos. São Carlos, 2006. Anais. São Carlos: EDUFSCar; 2007.

9. Pinheiro RC, Martinez CMS, Pamplin RCO. Suporte informativo para educadores de creche: risco e proteção nos primeiros anos de vida. Cad Ter Ocup UFSCar, São Carlos. 2010;18(2):129-38. Disponível em: http://www. cadernosdeterapiaocupacional.ufscar.br/index.php/cadernos/ article/view/348/288

10. Della Barba PCS, Joaquim RHVT, Martinez CMS, Jóia AF, Marini BPR, Pelissari DC, Lopes JF, Coronado NB. Ações conjuntas entre professores da educação infantil e alunos de terapia ocupacional: relato de experiência. Temas Desenvolv. 2013;19(105):120-4.

Recebido para publicação: 04.02.14

Aceito para publicação: 20.04.15
11. Law M, Missiuna C, Pollock N, Stewart D. Fundations for occupational therapy practice with children. In: Case-Smith J. Occupational therapy for children. St Louis: Elsevier; 2005.

12. Missiuna C, Pollock N, Egan M, Delaat D, Gaines R, Soucie $\mathrm{H}$. Enabling occupation through facilitating the diagnosis of developmental coordination disorder. Can J Occup Ther. 2008;75(1):26-34. doi: 10.2182/cjot.07.012.

13. Pinheiro RC. Coordenação viso motora e desenvolvimento global de crianças pré-termo: avaliação e detecção de riscos no início da escolarização [Dissertação]. São Carlos: Universidade Federal de São Carlos, Programa de PósGraduação em Terapia Ocupacional; 2012. Disponível em: http://www.bdtd.ufscar.br/htdocs/tedeSimplificado/tde busca/arquivo.php? codArquivo=4913.

14. Bardin L. Análise de conteúdo. 6a ed. Lisboa: Edições 70; 2011. 